\title{
Increased Inhibitor of Diifierentiation 4 (Id4) Expression in Glioblastoma: A Tissue Microarray Study
}

\author{
Weifin Zeng ${ }^{1}$, Elisabeth J. Rushing ${ }^{\circledR}{ }^{凶}$, Daniel P. Hartmann ${ }^{1}$, Norio Azumi ${ }^{1}$ \\ 1. Georgetown University Hospital, Washington, DC, USA \\ 2. Department of Neuropathology and Ophthalmic Pathology, Armed Forces Institute of Pathology, Washington, DC, USA
}

Corresponding author: Elisabeth J. Rushing, MD, Chair, Department of Neuropathology and Ophthalmic Pathology, Armed Forces Institute of Pathology, Washington, DC 20306-6000. Telephone: 202-782-3603; FAX: 202-782-4099; elisabeth.rushing@gmail.com

Published: 2010.06.01

\begin{abstract}
Background: The inhibitor of differentiation/DNA binding protein family (IdI-4) is involved in cell cycle control, tumorigenesis and angiogenesis through the negative regulation of helix-loop-helix transcription factors. Of these proteins, Id4 is known to play an important role in neural stem cell differentiation, and deregulation has been implicated in glial neoplasia. However, the expression and significance of Id 4 in astrocytomas has not been fully addressed. Herein we report the differential expression of Id4 in astrocytomas of various grades using tissue microarrays (TMA) and immunohistochemistry (IHC).

Design: The GBM TMA was constructed from 53 archival cases at Georgetown University Hospital and a TMA with normal brain controls and grades II-III astrocytoma was obtained from Cybrdi (Rockville, MD). TMA sections were stained with Id4 antibody and the slides were scored according to the percentage of staining astrocytic nuclei ( $<9 \%-, 10-50 \%+,>51 \%$ $++)$. The Fisher Exact test was used to test for statistical significance.

Results: Nuclear staining for Id4 was seen in $73.58 \%$ GBMs, $25 \%$ grade III, and I $2.5 \%$ grade II astrocytomas; staining was absent in normal brain tissue. There was a statistically significant difference between GBM and grades II, III astrocytoma $(p<0.0 \mathrm{I})$. Significant Id4 expression was not detected in normal brain.

Conclusions: Our study confirms the frequent upregulation of Id4 expression in GBM, which lends support to its role in tumorigenesis, possibly in the transformation of low to high-grade astrocytoma (i.e. GBM). Further studies are warranted to determine the precise role of Id 4 in glial neoplasia and its potential use in targeted therapy for GBM.
\end{abstract}

Key words: glioblastoma; helix-loop-helix; Id4; immunohistochemistry; tissue microarray; transcription factor

\section{Introduction}

Diffuse astrocytomas, World Health Organization (WHO) grades II-IV, are the most common primary brain tumors in adults [1]. Glioblastoma (grade IV), which comprises the majority, has a dismal prognosis with less than half of patients surviving more than one year. Early and extensive infiltration of neoplastic cells beyond their gross and radiographic appearance is a critical factor that limits the efficacy of surgery [2]. With rare exceptions, standard radio/chemotherapeutic regimens have been unable to obtain sustainable cures. Effective treatment of glial neoplasms is therefore an important unmet 
challenge and there is an urgent need for new therapies targeting the molecular and cellular mechanisms that mediate tumor growth.

Complex multigenetic pathways have been identified in the oncogenesis, progression, and maintenance of astrocytic brain tumors. Novel therapies that have the potential for overcoming limitations associated with currently available drugs are now in varying phases of clinical development [3]. Despite recent progress, major challenges remain in clarifying the biology of astrocytic tumors and translating basic research advances into effective therapies.

The inhibitor of differentiation protein family is involved in cell cycle control, tumor genesis and angiogenesis through the negative regulation of helix-loop-helix transcription factors [4-6]. To date, four structurally similar proteins (Id1-4) have been characterized that perform different regulatory functions during development, and when deregulated, during oncogenesis [7-11]. Of these proteins, Id4 has been reported to participate in neural stem cell differentiation, induce apoptosis in astrocytic cultures, promote angiogenesis, and drive brain tumor genesis through notch signaling [12-16]. However, the precise role and clinical relevance of Id4 in the formation and progression of gliomas remains elusive. Herein we report the differential expression of Id4 in astrocytomas of various grades including GBM using tissue microarrays (TMA) and immunohistochemistry (IHC) and discuss its possible role in astrocytoma oncogenesis.

\section{Materials and Methods}

This retrospective study consists of 53 cases of surgically resected GBM that were retrieved from the files of the Department of Pathology, Georgetown University Medical Center. All cases were reviewed and classified according to the 2007 World Health Organization (WHO) classification system [1]. For study purposes, a tissue microarray (TMA) was obtained from Cybrdi (Rockville, MD) that contained normal brain, and astrocytoma (grades II, III) tissue cores. Specifically, the array had a total of 32 cores with 8 grade II and 8 grade III astrocytomas, and 16 cores of normal brain tissue.

\section{Glioblastoma tissue microarray construction}

Areas showing the histopathologic features of GBM were selected on archival H\&E slides, and then, representative areas of tumor were marked on the corresponding paraffin block for TMA construction. Briefly, after the tissue cylinders were taken from the selected regions of the donor paraffin block, they were then punched precisely into a recipient paraffin block using a tissue-arraying instrument. Multiple sections ( $5 \mu \mathrm{m}$ thick) were cut and mounted onto microscope slides. An H\&E stained section was made to confirm the presence of the original areas selected from each tumor. The TMA sections were evaluated by two pathologists in a blinded fashion to validate the diagnostic morphology of each array spot. All images were taken using an Olympus BX41 microscope.

\section{Immunohistochemistry}

TMA sections were stained with Id4 antibody (1:100, Chemicon International, Temecula, CA) using a DAKO autoimmunostainer and Envision Flex detection system (DAKO, Carpinteria, CA). The immunostaining pattern (i.e., cytoplasmic, nuclear, membranous) for Id 4 were assessed by a semiquantitative method within the neoplastic cell and vascular components. Adequate immunoreactive tissue samples were used as positive controls for each antibody. Negative controls were produced by omission of the primary antibody.

\section{Statistical analysis}

The Fisher exact test was carried out to evaluate the strength of association between the presence of Id4 staining and each tumor subset (glioblastoma, anaplastic astrocytoma, grade III and astrocytoma, grade II). Values of $P<0.05$ were considered statistically significant.

\section{Results}

\section{Interpretation of immunohistochemical staining}

A summary of the immunohistochemical results is presented in Table 1.

Table I Summary of Id4 Immunostaining results

\begin{tabular}{llc}
\hline Diagnosis & No. of TMA cores $\%$ Nuclear staining \\
\hline Normal & 16 & $0 / 16(0 \%)$ \\
\hline Astrocytoma, grade II & 8 & $1 / 8(12.5 \%)$ \\
\hline Astrocytoma, grade III & 8 & $2 / 8(25.0 \%)$ \\
\hline Glioblastoma & 53 & $39 / 53(73.6 \%)$ \\
\hline
\end{tabular}

The extent of staining was scored semiquantitatively by estimating the average percentage of staining nuclei in tumor cells $(<9 \%=$ negative, $10-50 \%$ $=$ positive, $>51 \%++$ ). Only cells with moderately intense nuclear staining were interpreted as positive for Id4. Significant cytoplasmic or membranous staining was not observed. Neoplastic astrocytes in GBM showed intense nuclear immunoreactivity in 39 of 53 (73.58\%), whereas 2 of $8(25 \%)$ grade III and 1 of 8 $(12.5 \%)$ of grade II astrocytomas showed staining (Figure 1). 


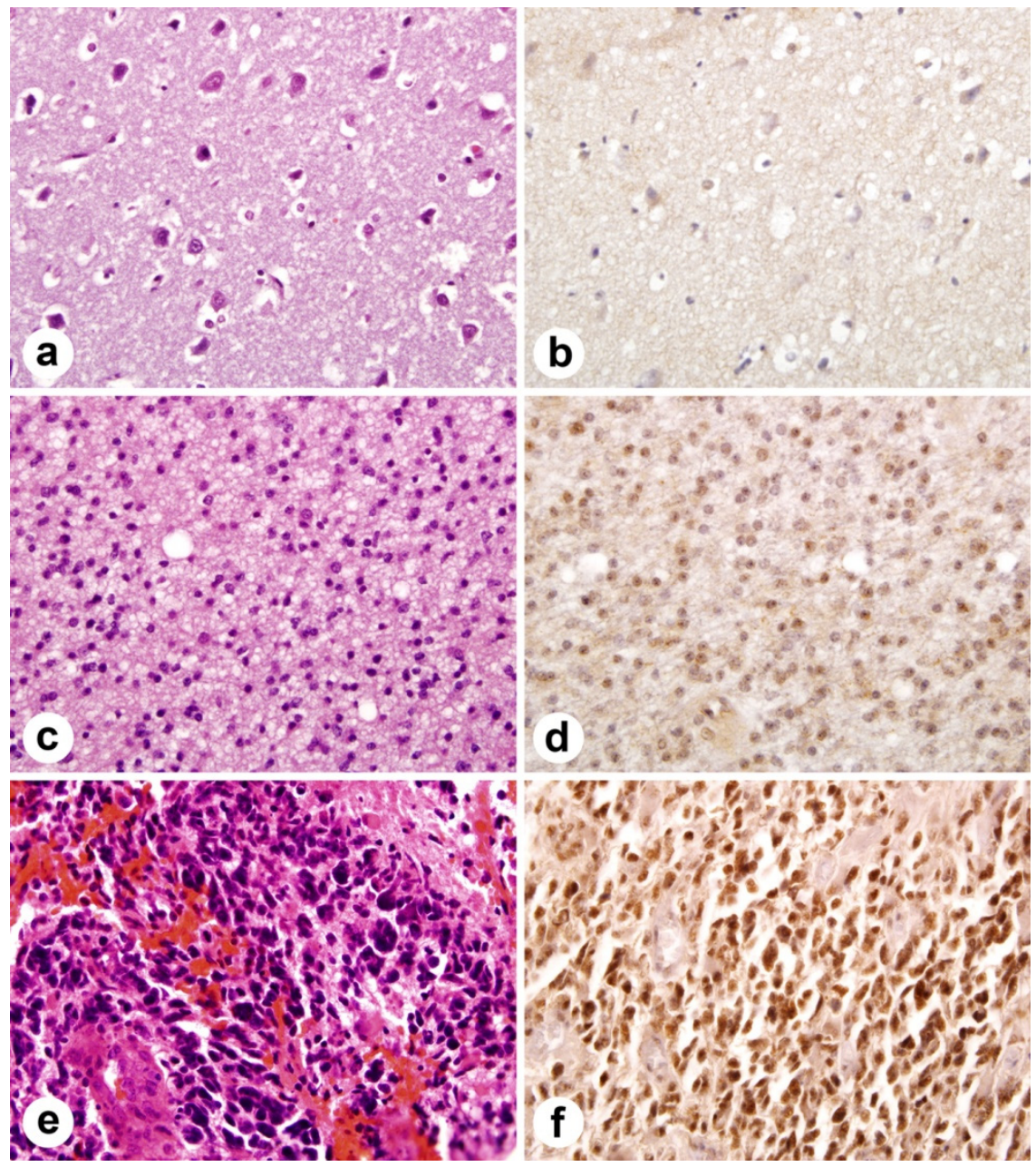

Figure I. Immunohistochemical staining of Id4 protein in tissue microarray. Id4 expression correlates with astrocytoma grade. (a) normal brain tissue (H\&E). (b) normal brain tissue lacks Id4 staining. (c) moderately cellular grade II astrocytoma with nuclear pleomorphism (H\&E). (d) note scattered nuclei immunolabel for Id4 in grade II astrocytoma. (e) GBM (H\&E) with marked nuclear pleomorphism and microvascular proliferation. (f). widespread nuclear staining in GBM.

Endothelial cell nuclei within tumor blood vessels were devoid of significant Id4 labeling and only low-level background staining was seen in normal brain tissue cores $(0 / 16)$.

\section{Statistical analysis}

There was a statistically significant difference between GBM and normal brain tissue, GBM and grade II, III astrocytoma ( $\mathrm{p}<0.01)$. No statistically significance was detected between normal brain tissue, grade II and grade III astrocytoma ( $p>0.05)$.

\section{Discussion}

In this study, we documented a marked increase in expression of Id4 in GBM compared to low-grade astrocytoma. While significant staining was not detected in normal brain or in tumor blood vessels; we observed a potentially clinically relevant strong cor- relation between the nuclear expression of Id4 and histological grade, with the highest expression seen in GBM (73.58\%) and anaplastic astrocytoma (25\%). The commercially available anti-Human Id4 monoclonal antibody that was used in this study was developed in mice and appears suitable for use in formalin-fixed paraffin-embedded tissue. Similar nuclear localization of Id4 in tumor cells has been reported in high-grade prostate carcinoma and small cell lung carcinoma $[17,18]$. The selective nuclear localization of Id4 in glial tumors contrasts with the dual cytoplasmic-nuclear expression of Id1-3. Of note, Vandeputte et al. demonstrated that Id1-3 displayed cytoplasmic as well as nuclear localization and that expression was positively correlated with proliferation indices [19].

It is not surprising that Id proteins are implicated in oncogenesis. Since their expression correlates in- 
versely with cell differentiation, it has been postulated that these proteins participate in the activation of transcription factors that stimulate reprogramming of the cells into cancer stem-like cells [11-12]. Along parallel lines, Marin-Hustegge detailed the complex role of Id4 as an oligodendrocyte differentiation inhibitor with the ability to differentially regulate the expression and subcellular distribution of myelin gene products [20].

With respect to other members of the Id family, Id 2 has been shown to mediate tumor initiation, proliferation, and angiogenesis In Rb mutant mice [19]. In humans, Id 2 expression may play a role in the malignant progression of small cell lung carcinoma that may be prognostically significant [18]. Forootan et al. reported that Id-1 overexpression in prostate cancer was associated with reduced patient survival [22]. Other investigators have identified a similar predictive value for Id proteins in breast [23], colorectal [24] and bladder cancer [25], further underscoring their importance in neoplasia in multiple organs.

Although the precise mechanism of Id4 in promoting glial neoplasia remains incompletely characterized, several mechanisms have emerged in recent studies. Most recently, Kuzontkoski et al. reported that Id4 promotes angiogenesis and growth of GBM by elevating matrix GLA levels [14]. In an earlier study, Fontemaggi et al. identified Id4 as part of a transcriptional axis, including mutant p53 and E2F1 that contributed to tumor neo-angiogenesis [15]. Earlier studies by Jeon et al. indicated that Id4 drives brain tumor-initiating cell genesis through cyclin $\mathrm{E}$ and notch signaling [16]. Interestingly, Liang et al. observed that Id4 transcripts were present in both GBM and oligodendrogliomas, whereas protein expression of Id 4 was present in reactive and neoplastic astrocytes, but not in neoplastic oligodendrocytes [26]. This selective expression of Id4 in astrocytes requires further investigation to determine whether it might be exploited for diagnostic purposes.

In addition to enhancing our understanding of key events in the development of primary brain tumors, Ig4 immunolabeling may provide a pragmatic diagnostic tool. For the surgical pathologist, differentiating glioblastoma from low grade gliomas or low-grade gliomas from gliosis is usually straightforward based on histologic features; however, it can be problematic, particularly in the setting of small biopsy specimens. In addition, as noted above, Id4 immunohistochemistry may provide useful information for distinguishing oligodendroglial from astrocytic tumors. Accordingly, the confirmation of Id4 as a signature biomarker has the potential to increase our diagnostic acumen for this lethal disease.
In conclusion, our study confirms that Id4 immunohistochemistry has a high overall expression in GBM, but is less efficacious in separating grade II from grade III astrocytoma. Although these results are promising, its limitations should be kept in mind when considering Id4 immunohistochemistry for diagnostic purposes. The experience with this antibody is still limited and its expression in other glial neoplasms and other tumors that can mimic high grade astrocytomas has not been systematically studied. Based on these findings, additional studies are warranted before the biologic, prognostic or therapeutic role of ID4 expression can be firmly established.

\section{Conflict of Interest}

The authors have declared that no conflict of interest exists.

\section{References}

1. Kleihues P, Burger PC, Aldape KD, et al. DD Glioblastoma. In: Louis DN, Ohgaki H, Wiestler OD and Cavenee WK, eds. WHO classification of tumors of the central nervous system. Lyon: IARC Press. 2007:33-49.

2. Sathornsumetee S, Reardon DA, Desjardins A et al. Molecularly targeted therapy for malignant glioma. Cancer. 2007;110:13-24.

3. Rutka JT, Kongkham P, Northcott $P$ et al. The evolution and application of techniques in molecular biology to human brain tumors: a 25 year perspective. J Neurooncol. 2009;92:261-73.

4. Norton JD. ID helix-loop-helix proteins in cell growth, differentiation and tumorigenesis. J Cell Sci. 2000;113:3897-905.

5. Zebedee Z, Hara E. Id proteins in cell cycle control and cellular senescence. Oncogene 2001;20:8317-25.

6. Iavarone A, Lasorella A. ID proteins as targets in cancer and tools in neurobiology. Trends Mol Med 2006;12:588-94.

7. Ruzinova MB, Benezra R. Id proteins in development, cell cycle and cancer. Trends Cell Biol 2003;13:410-8.

8. Langlands K, Yin X, Anand G, et al. Differential interactions of Id proteins with basic-helix-loop-helix transcription factors. J Biol Chem. 1997;272:19785-93.

9. Zhu W, Dahmen J, Bulfone A, et al. Id gene expression during development and molecular cloning of the human Id-1 gene. Brain Res Mol Brain Res. 1995; 30:312-26.

10. Andres-Barquin PJ, Hernandez MC, Israel MA. Id genes in nervous system development. Histol Histopathol. 2000;15:603-18.

11. Duncan M, DiCicco-Bloom EM, Xiang X, et al. The gene for the helix-loop-helix protein, Id, is specifically expressed in neural precursors. Dev Biol. 1992;154:1-10.

12. Yun K, Mantani A, Garel S, et al. Id4 regulates neural progenitor proliferation and differentiation in vivo. Development. 2004;131:5441-8.

13. Andres-Barquin PJ, Hernandez MC, Israel MA. Id4 expression induces apoptosis in astrocytic cultures and is down-regulated by activation of the cAMP-dependent signal transduction pathway. Exp Cell Res. 1999;247:347-55

14. Kuzontkoski PM, Mulligan-Kehoe MJ, Harris BT, et al. Inhibitor of DNA binding-4 promotes angiogenesis and growth of glioblastoma multiforme by elevating matrix GLA levels. Oncogene. 2010; [Epub ahead of print].

15. Fontemaggi G, Dell'Orso S, Trisciuoglio D, et al. The execution of the transcriptional axis mutant p53, E2F1 and ID4 promotes tumor neo-angiogenesis. Nat Struct Mol Biol. 2009;16:1086-93. 
16. Jeon HM, Jin $X$, Lee JS, et al. Inhibitor of differentiation 4 drives brain tumor-initiating cell genesis through cyclin $\mathrm{E}$ and notch signaling. Genes Dev. 2008; 22:2028-33.

17. Yuen HF, Chua CW, Chan YP, et al. Id proteins expression in prostate cancer: high-level expression of Id-4 in primary prostate cancer is associated with development of metastases. Mod Pathol 2006;19:931-41.

18. Kamalian L, Gosney JR, Forootan SS, et al. Increased expression of Id family proteins in small cell lung cancer and its prognostic significance. Clin Cancer Res. 2008;14:2318-25.

19. Vandeputte DA, Troost D, Leenstra S, et al. Expression and distribution of id helix-loop-helix proteins in human astrocytic tumors. Glia. 2002;38:329-38.

20. Marin-Husstege M, He Y, Li J, et al. Multiple roles of Id4 in developmental myelination: predicted outcomes and unexpected findings. Glia. 2006;54:285-96.

21. Lasorella A, Rothschild G, Yokota Y, et al. Id2 mediates tumor initiation, proliferation, and angiogenesis in $\mathrm{Rb}$ mutant mice. Mol Cell Biol. 2005; 25:3563-74.

22. Forootan SS, Wong YC, Dodson A, et al. Increased Id-1 expression is significantly associated with poor survival of patients with prostate cancer. Hum Pathol. 2007; 38:1321-9.

23. Noetzel E, Veeck J, Niederacher D, et al. Promoter methylation-associated loss of ID4 expression is a marker of tumour recurrence in human breast cancer. BMC Cancer. 2008; 8:154

24. Wilson JW, Deed RW, Inoue T, et al. Expression of Id helix-loop-helix proteins in colorectal adenocarcinoma correlates with p53 expression and mitotic index. Cancer Res. 2001; 61:8803-10.

25. Ding Y, Wang G, Ling MT, et al. Significance of Id-1 up-regulation and its association with EGFR in bladder cancer cell invasion. Int J Oncol. 2006;28(4):847-54.

26. Liang Y, Bollen AW, Nicholas MK, et al. Id4 and FABP7 are preferentially expressed in cells with astrocytic features in oligodendrogliomas and oligoastrocytomas. BMC Clin Pathol. 2005;5:6. 